\title{
Cases of Sudden Death in a Sheep Model Implanted with Aortic Bioprostheses
}

\begin{abstract}
Keywords: Bioprosthetic heart valves; Aortic stenosis; Heat-stress; Sheep

Abstract

Background: Sheep have long been established as an anima model in heart valve research for the preclinical safety assessment of prosthetic and bioprosthetic cardiac valves. Complic ations unrelated to the implant or surgical procedure are uncommon, but do represent a significant loss of data when they occur. Here we report the pre-term deaths of 3 sheep implanted with a bioprosthetic aortic valve.

Aims: (1) To present potential physiological limitations of bioprosthetic aortic valves and the effect on the host animals' ability to compensate for environmental stressors and (2) consider these findings as they relate to the future long-term management of these animals during the in-life stage of studies.

Methods: The early deaths of 3 sheep implanted with a nove bioprosthetic aortic valve were retrospectively analyzed including medical records, surgical records, pathology, and historical weather data.

Results: Necropsy of all sheep revealed a stenotic left ventricular outflow tract and general findings consistent with acute congestive heart failure as a cause of death secondary to heat stress. Historical weather data identified high ambient temperatures, low wind speeds, and moderate humidity on the days the sheep died.

Conclusion: The placement of bioprosthetic valve in the aortic position in sheep can result in significant compromise to the left side of the heart. It should be assumed that these sheep have a decreased cardiac reserve, limited ability to compensate in response to environmenta stressors, and are at increased risk of adverse cardiac events including those possible during a heat wave. This should be considered when determining the appropriate post operative care of these animals.
\end{abstract}

\section{Introduction}

Sheep have long been established as an animal model in heart valve research for the preclinical safety assessment of prosthetic and bioprosthetic cardiac valves [1-3]. The cardiovascular system and blood coagulation profile of sheep is similar to humans and the aortic root allows for implantation and evaluation of cardiac devices using standard cardiopulmonary bypass and anesthetic protocols [1]. In addition, sheep are relatively easily maintained, compared to other species, and exhibit the temperament and handling characteristics conducive to long-term evaluation [1].

To demonstrate the preclinical in vivo safety of bioprosthetic valves, studies are conducted for a minimum of 20 weeks per ISO standard 5840 [4]. For the portion of the study that does not involve surgical procedures or diagnostic testing, our lab houses sheep at a farm-based research facility for long-term postoperative care. In the authors' opinion this decreases daily stress and improves animal welfare.

It is the authors' experience that pre-term deaths of sheep implanted with bioprosthetic heart valves occur at low to medium frequency. Most pre-term deaths are the result of rapid calcification
Journal of Veterinary Science \& Medicine

Stephen Q Garofolo ${ }^{1 *}$, Christine Watson ${ }^{2}$, Richard W Bianco ${ }^{1}$ and Nicholas A Robinson ${ }^{1,2}$

${ }^{1}$ Experimental Surgical Services, University of Minnesota Medical School, USA

${ }^{2}$ University of Minnesota College of Veterinary Medicine, USA

\section{Address for Correspondence}

Stephen Q Garofolo, DVM, MMC 220 Mayo, 8220A, 420 Delaware St. SE, Minneapolis, MN 55455, Tel: +1 561370 8844; Fax: +1 612626 0942; Email: sgarofol@umn.edu

Copyright: (c) 2014 Garofolo SQ, et al. This is an open access article distributed under the Creative Commons Attribution License, which permits unrestricted use, distribution, and reproduction in any medium, provided the original work is properly cited.

Submission: 12 August 2014

Accepted: 18 August 2014

Published: 22 August 2014

Reviewed \& Approved by: Dr. Mark Brown, Professor and faculty in the Department of Clinical Sciences at Colorado State University, USA

of leaflets, structural valvular dysfuction, paravalvular leaks, or other surgical complications leading to congestive heart failure and death. Early deaths unrelated to the valve or surgical procedure, such as environmental stress or natural disease are not common, and while precautionary steps in the husbandry and management of the animals are taken to prevent them, they do occur occasionally. These deaths represent a significant loss of data and can be detrimental to the outcome of the study.

The current case series describes the pre-term deaths of 3 female sheep implanted with a novel bioprosthetic aortic valve. The major underlying factor resulting in cardiac decompensation and death of these animals was considered to be excessive heat load. The specific aims of this paper are to (1) present potential physiological limitations of bioprosthetic aortic valves and the effect on the host animals' ability to compensate for environmental stressors (i.e. heat and transport) and (2) consider these findings as they relate to future long-term management of these animals during the in-life stage of studies.

\section{Material and Methods}

In April of 2012, 19 adult Polypay sheep were enrolled in a study for the evaluation of a novel aortic bioprosthesis. The sheep were examined by university staff veterinarians, determined to be in normal health, and vaccinated for Clostridium perfringens Type D, Clostridium tetani, and Corynebacterium pseudotuberculosis. All of the sheep were acclimated to the new housing for at least 3 days before the respective date of surgery.

The sheep were implanted with a prosthetic aortic valve as previously described [5]. The device was placed in the supraannular position through a left thoracotomy with the animal on cardiopulmonary bypass. 10 of the sheep received a novel aortic bioprosthetic (test article) and 9 received a commercially available aortic bioprosthetic valve (control article).

Post-operatively, the animals were examined and treated daily by 
Citation: Garofolo SQ, Watson C, Bianco RW, Robinson NA. Cases of Sudden Death in a Sheep Model Implanted with Aortic Bioprostheses. J Veter Sci Med. 2014;2(2): 4.

veterinary staff. All sheep were treated with heparin (Hospira, Inc. Lake Forest, IL) 2000 IU SQ SID on the day of surgery, then BID for two days, ceftiofur sodium (Pfizer Inc., New York, NY) $2 \mathrm{mg} / \mathrm{kg}$ IM for 8 days, buprenorphine (Rekitt Benckiser Healthcare Hull, England) $0.03 \mathrm{mg} / \mathrm{kg}$ IM PRN for pain, fentanyl (Mallinckrodt Inc. Hazelwood, MO) TD for 72 hours, and ketoprofen (Fort Dodge Animal Health, Fort Dodge, IA) $1-2 \mathrm{mg} / \mathrm{kg}$ IM PRN for pain. At 14 days postoperatively trans-thoracic echocardiography was performed to acquire routine data measurements. At this time the sheep were examined by University of Minnesota staff veterinarians. All of the sheep were cleared for transfer, and subsequently transported to a lay-up farm for long term post-operative care.

At the farm the sheep were housed in an outdoor paddock with available shelter from the sun and free access to clean fresh water. They were observed twice daily for clinical signs of pain and distress by a certified veterinary technician. A local veterinarian was on call for required medical treatment and/or consultation.

Between May 19th and May 27th, 2012, 3 of the sheep suffered early deaths prior to the end of the study (Table 1). Sheep 1 died during transport to the farm. Sheep 2 was found dead in the outdoor paddock 4 days after arrival. Sheep 3 was found dead in the outdoor paddock 3 days after arrival. No other animals on the farm died during this time, including horses, dogs, pigs, and other sheep implanted with prosthetic heart valves.

Necropsies were performed on the sheep within 12 hours of death. A gross examination of all organ systems including the device was conducted. Histological examination of gross lesions found during necropsy and also of the device was performed. Tissues were fixed in $10 \%$ neutral buffered formalin before being routinely processed and stained with hematoxylin and eosin. Historical weather data for western Wisconsin from May 19th to May 27th 2012 was obtained. Study data including surgical, medical records, and diagnostic imaging was reviewed. The research study was conducted with approval of the University of Minnesota IACUC committee.

\section{Results}

\section{Surgical and post-operative data}

Intra-operative measurements of the internal diameter of the native aortic annulus were $20 \mathrm{~mm}, 19 \mathrm{~mm}$, and $19 \mathrm{~mm}$ for sheep $1-3$ respectively (Mean $19.3 \mathrm{~mm}+/-0.58$ ). Sheep 1 and 2 received the test article and Sheep 3 received the control article. All of the valves implanted had an internal diameter of $15.4 \mathrm{~mm}$, per product specifications from the manufacturer. No surgical complications were reported and all of the sheep recovered without incident.

Day 14 echocardiography findings in the 3 sheep were comparable to data obtained from other sheep in the study that went to term, data for sheep 1-3 is reported in Table 2, including maximum and mean transvalvular pressure gradient, effective orifice area, and heart rate. Mean values for all of the sheep in the study are reported in Table 3.

Furosemide (Ranbaxy Pharmaceuticals Inc., Jacksonville, FL) 4 $\mathrm{mg} / \mathrm{kg}$ was administered IM to sheep 1 and 3 once, on the day of surgery post-operatively based on clinical signs of pulmonary edema. Physical exam prior to transport revealed a heart murmur in sheep 1 and muffled heart sounds in sheep 3. No other clinical signs of cardiovascular disease were noted. All of the sheep were cleared for transfer by the attending veterinarian.

All sheep were identified as having stable vital parameters the day after arriving at the farm. No additional observations were noted until the time of death.

\section{Historical weather data}

Historical weather data from western Wisconsin from May 19th to May 27th reported a maximum temperature of $91^{\circ} \mathrm{F}$ and a minimum temperature of $56^{\circ} \mathrm{F}$ [6]. On the days the ewes died, there were high ambient temperatures, low wind speeds, and moderate humidity (Table 4).

\section{Pathology}

The internal orifice diameter of the inflow aspect of the device post-mortem was severely reduced at $11 \mathrm{~mm}, 12 \mathrm{~mm}$, and $13 \mathrm{~mm}$, for sheep $1-3$ respectively (Mean $12+/-1 \mathrm{~mm}$ ) (Table 1 ). There were no changes in any of the gross cardiac measurements taken post mortem. Microscopically, abdundant fibrocellular pannus was present at the inflow and outflow aspects of each of the 3 prosthetic valves. Sheep 2 had a marked amount of fibrocellular pannus extending over the valve frame post and into the commissures of the prosthetic leaflets. Mild thrombosis of all leaflets was identified in sheep 1 and 3. Sheep 2 had moderate thrombosis of both the inflow and outflow aspects of the valve leaflets. General findings in all 3 sheep were related to varying degrees of advanced internal autolysis inconsistent with the

Table 1: Subject Data- Table reports age, type of implant, duration of implant, circumstances of death, and internal diameter of the inflow aspect of the implanted valve for each sheep that suffered an early death.

\begin{tabular}{|c|c|c|c|c|c|}
\hline Subject & Age (yrs.) & Implant & Duration of Implant & $\begin{array}{c}\text { Int. Diameter Inflow } \\
\text { post-mortem (mm) }\end{array}$ & Transport \\
\hline 1 & 4 & Test $19 \mathrm{~mm}$ & 29 days & Found Dead & 12 \\
\hline 2 & 2 & Test $19 \mathrm{~mm}$ & 22 days & Found Dead & 13 \\
\hline 3
\end{tabular}

Table 2: Echo Data-Table reports echo data at day 14 time point for sheep 1, 2, and 3.

\begin{tabular}{|c|c|c|c|c|c|}
\hline Subject & $\begin{array}{c}\text { Max Pressure } \\
\text { Gradient (mmHg) }\end{array}$ & $\begin{array}{c}\text { Mean Pressure } \\
\text { Gradient (mmH) }\end{array}$ & $\begin{array}{c}\text { Cardiac Output } \\
\text { (L/min) }\end{array}$ & $\begin{array}{c}\text { Effective Orifice } \\
\text { Area (cm2) }\end{array}$ & $\begin{array}{c}\text { Heart Rate (bpm) } \\
1.2\end{array}$ \\
\hline 1 & 69 & 44 & 10.6 & 136 \\
\hline 2 & 76 & 50 & 13.5 & 1.27 \\
\hline 3 & 71 & 42 & 10.8 & 1.0 \\
\hline
\end{tabular}


Citation: Garofolo SQ, Watson C, Bianco RW, Robinson NA. Cases of Sudden Death in a Sheep Model Implanted with Aortic Bioprostheses. J Veter Sci Med. 2014;2(2): 4.

ISSN: $2325-4645$

Table 3: Echo Data (Mean values) -Table reports mean echo values for all animal subjects enrolled in the study.

\begin{tabular}{|c|c|c|c|c|}
\hline Time Point & $\begin{array}{c}\text { Max Pressure } \\
\text { Gradient (mmHg) }\end{array}$ & $\begin{array}{c}\text { Mean Pressure } \\
\text { Gradient (mmHg) }\end{array}$ & $\begin{array}{c}\text { Cardiac Output } \\
\text { (L/min) }\end{array}$ & $\begin{array}{c}\text { Effective Orifice } \\
\text { Area (cm2) }\end{array}$ \\
\hline $\begin{array}{c}\text { Control 14 day } \\
\text { mean }\end{array}$ & 61.92 & 38.00 & 9.93 & 1.12 \\
\hline $\begin{array}{c}\text { Test 14 day } \\
\text { mean }\end{array}$ & 75.70 & 48.20 & 9.66 & 141.17 \\
\hline
\end{tabular}

Table 4: Historical Weather Data-Table reports the temperature, relative humidity, and wind speed on the days the sheep died.

\begin{tabular}{|c|c|c|c|c|}
\hline Subject & $\begin{array}{c}\text { Date of } \\
\text { Death }\end{array}$ & Max - Min Temp ( ${ }^{\circ}$ F) & Relative Humidity (\%) & $66-41$ \\
\hline 1 & $5 / 24 / 2012$ & $82-56$ & $60-26$ \\
\hline 2 & $5 / 19 / 2012$ & $91-63$ & 13 \\
\hline 3 & $5 / 27 / 2012$ & $89-65$ & $87-50$ \\
\hline
\end{tabular}

deterioration of the external body. Sheep 2 had diffuse autolysis of all organs. Sheep 1 and 3 contained voluminous, foam filled lungs consistent with pulmonary edema. Small abscesses were identified on the heart base, gastrointestinal tract, and mammary glands of sheep 1 (consistent with local infection with Corynebacterium pseudotuberculosis), as well as significant cardiomyocyte atrophy and fibrosis in the left ventricle, but no change in left ventricular free wall thickness.

\section{Discussion}

Acute congestive heart failure secondary to heat stress is considered to be the cause of death of these 3 sheep. Ambient temperatures and humidity above normal in combination with necropsy findings consistent with heat related injury strongly suggests that heat stress was the inciting cause of heart failure. Additional support is provided by clinical signs as well as the presence of a stenotic left ventricular outflow tract with secondary pulmonary edema. The authors' assume that all healthy sheep implanted with a prosthetic aortic heart valve have diminished cardiac capacity and are at increased risk of adverse cardiac events when exposed to elevated temperatures. This critical limitation following implantation should be considered in the longterm post-operative management of sheep implanted with novel aortic bioprostheses.

The identification of a grade III/VI left sided systolic murmur in one of the sheep suggests the presence of aortic stenosis prior to transport. The placement of an aortic prosthesis in the native annulus elicited thrombosis and pannus formation, which markedly reduced the diameter of the left ventricular outflow tract. The intra-operative measurements of the native aortic annulus in the 3 sheep had a mean internal diameter of $19.3 \mathrm{~mm}+/-0.58$. The placement of a $19 \mathrm{~mm}$ (total diameter) prosthetic aortic valve with an internal orifice diameter of $15.4 \mathrm{~mm}$ resulted in a significant loss in effective area of the left ventricular outflow tract. This area was further decreased with the addition of thrombosis and pannus, as indicated by a mean internal diameter of $12 \mathrm{~mm}+/-1$ at postmortem examination in this study. This information in combination with pulmonary edema identified during necropsy supports the presence of aortic stenosis and acute congestive heart failure as the cause of death.

High ambient temperatures, moderate humidity, and low wind speeds at the time of death combined with rapid autolysis postmortem increase the likelihood that heat stress directly contributed to the death of these animals. Humidity and low wind speed are known to decrease the ability of mammals to dissipate heat [7]. Advanced internal autolysis that does not correlate with the decomposition level of the external body is a common finding in heat related injury [8] Most post-mortem findings in animals that succumb to heat stroke also include evidence of multiorgan dysfuction i.e. diffuse hemorrhage and necrosis [8,9]. It is suggested that these sheep died from congestive heart failure before the full effects of heat stroke, therefore these additional signs were absent. This is in contrast to a clinically normal animal that died from heat stroke.

Sheep experience heat stress when they are exposed to temperatures greater than $86^{\circ} \mathrm{F}$ [10]. They respond by increasing their respiratory rate and heart rate, as well as increasing blood flow to tissues important in heat exchange including legs, ears, and nasal mucosa while decreasing blood flow to other organs such as the spleen, kidneys, and gastrointestinal tract [11]. This integrated response to heat increases workload on the left ventricle [12]. In the current case of these 3 sheep, the exposure to excess heat would have caused an increase in heart rate in an attempt to maximize heat dissipation and maintain perfusion in the face of vasodilation [11]. The presence of a stenotic left ventricular outflow tract would have effectively reduced stroke volume limiting this to an inadequate response. Prolonged exposure to heat and continued stress on a compromised left ventricle unable to compensate led to acute left-sided congestive heart failure and ultimately death.

Similar responses have been observed in humans. Epidemiological studies report an increase in both morbidity and mortality during a heat wave [12]. Most of these events are not directly heat-related but instead cardiovascular in origin, brought about by the increased cardiovascular challenge associated with thermoregulatory responses to heat stress [12]. Of the large number of deaths in the 1995 Chicago heat wave, $93.7 \%$ were attributed to underlying cardiovascular disease [13]. It has also been shown that people with ventricular dysfunction and congestive heart failure have a reduced ability to dissipate thermal load due to a limited cardiac output reserve and attenuated cutaneous vasodilation $[14,15]$. Greater heat storage further exacerbates the strain on an already compromised cardiovascular system and could increase the likelihood of an adverse cardiac event [12]. Attenuated vasodilation (increased peripheral resistance) could have contributed to the cause of death in these sheep by increasing afterload and limiting heat dissipation.

To the best of our knowledge, specific guidelines for the 
Citation: Garofolo SQ, Watson C, Bianco RW, Robinson NA. Cases of Sudden Death in a Sheep Model Implanted with Aortic Bioprostheses. J Veter Sci Med. 2014;2(2): 4.

ISSN: 2325-4645

appropriate post-operative care of sheep after prosthetic heart valve replacement have not been published. Sheep are most comfortable in naturally structured outdoor enclosures with pasture[16], therefore our lab relocates sheep to a farm as soon as they are considered stable enough for transport, to decrease stress and improve animal welfare. In light of the most recent findings, strict adherence to this protocol should be reconsidered. In the authors' opinion, the risk of heat stress should outweigh the need to maintain sheep in a more natural environment in some cases. Some guidelines for the postoperative management of human patients with prosthetic heart valves discourage walking outside when the ambient temperature is above $80^{\circ} \mathrm{F}$ [17]. Similar recommendations could be made in sheep implanted with prosthetic heart valves.

It is possible that the sheep died from congestive heart failure alone, completely independent of heat stress, however this is considered unlikely based primarily on climatic and necropsy data. It should also be noted that the 15 sheep that survived to term in this study had similar hemodynamic parameters and internal diameters of the prosthetic valve post-mortem. Therefore, a more likely explanation includes heat stress as a major complicating factor. To confirm this hypothesis, controlled studies comparing the physiologic response of sheep implanted with prosthetic aortic valves and normal sheep to heat stress would be necessary, but ethically questionable. In the case of sheep 1 the sympathetic response to the stress of transport and handling was most likely an additional contributing factor.

\section{Conclusion}

In conclusion, although the deaths of the 3 ewes in this study were likely multifactorial, the placement of a bioprosthetic valve in the aortic position in sheep can result in significant compromise to the left side of the heart. It should be assumed that these sheep have a decreased cardiac reserve, limited ability to compensate in response to environmental stressors, and are at increased risk of adverse cardiac events including those possible during a heat wave. This should be considered when determining the appropriate post operative care of these animals.

\section{References}

1. Grehan JF, Casagrande I, Oliveira EL, Santos PC, Pessa CJ et al. (2001) A juvenile sheep model for the long-term evaluation of stentless bioprotheses implanted as aortic root replacements. J Heart Valve Dis 10: 505-512.
2. Rakow N, Nelson DA, Falkner P, Wahlberg P, Grangaard R et al. (2007) Orthotopic Total Aortic Root Replacement Model in Adult Sheep. J Invest Surg 20: 55-59.

3. Trantina-Yates A, Weissenstein C, Human P, Zilla P (2001) Stentless Bioprosthetic Heart Valve Research: Sheep Versus Primate Model. Ann Thorac Surg 71: S421-S427.

4. ANSI/AAMI/ISO 5840: 2005. Cardiovascular implants-cardiac valve prostheses. Arlington, VA: Association for the Advancement of Medical Instrumentation; 2005.

5. Ouyang DW, Salerno CT, Pederson TS, Bolman RM 3rd, Bianco RW (1998) Long-term evaluation of orthotopically implanted stentless bioprosthetic aortic valves in juvenile sheep. J Invest Surg 11: 175-183.

6. Historical Weather Data Eau Claire, Wisconsin, USA [Internet] 2012 May [cited 2014 June 12].

7. Sula MM, Winslow CM, Boileau MJ, Barker LD, Panciera RJ (2012) Heatrelated injury in lambs. J VET Diagn Invest 24: 772-776.

8. Merck MD (2012) Veterinary Forensics: Animal Cruelty Investigations. 2nd Ed. Wiley-Blackwell (online).

9. Bruchim Y, Loeb E, Saragusty J, Aroch I (2009) Pathological Findings in Dogs with Fatal Heatstroke J Comp Path 140: 97-104.

10. Alhidary IA, Shini S, Al Jassim RAM, Gaughan JB (2012) Physiological Responses of Australian Merino Wethers Exposed to High Heat Load. J Anim Sci 90: 212-220.

11. Hales JRS (1973) Effects of Exposure to Hot Environments on the regional distribution of blood flow and on cardiorespiratory function in sheep. Pflugers Arch 344: 133-148.

12. Kenney WL, Craighead DH, Alexander LM (2014) Heat Waves, Aging and Human Cardiovascular Health. Med Sci Sports Exerc.

13. Kaiser R, Le Tertre A, Schwartz J, Gotway CA, Daley WR, et al. (2007) The effect of the 1995 heat wave in Chicago on all-cause and cause-specific mortality. Am J Public Health 97: Suppl 1: S158-S162.

14. Cui J, Sinoway LI (2014) Cardiovascular Responses to Heat Stress in Chronic Heart Failure. Curr Heart Fail Rep 11: 139-145.

15. Cui J, Arbab-Zadeh A, Prasad A, Durand S, Levine BD, et al. (2005) Effects of Heat Stress on Thermoregulatory Responses in Congestive Heart Failure Patients. Circulation 112: 2286-2292.

16. Reinhardt V, Reinhardt A (2002) Comfortable quarters for sheep in research institutions. In: Reinhardt V, Reinhardt A, eds. Comfortable Quarters for Laboratory Animals. (9th edition) Animal Welfare Institute, Washington, DC.

17. Patient guide to Heart Surgery. [online handout] (2013) Mountain View Hospital, Las Vegas, NV. 